\title{
Strong field processes inside gallium arsenide
}

\author{
S M Golin ${ }^{1,2}$, S E Kirkwood ${ }^{1}$, D D Klug ${ }^{3}$, D M Villeneuve ${ }^{3}$, D M Rayner ${ }^{3}$, \\ C A Trallero Herrero ${ }^{1,2}$ and $\mathbf{P}$ B Corkum ${ }^{1}$
}

\author{
${ }^{1}$ Joint Attosecond Science Laboratory, National Research Council and University of Ottawa, 100 Sussex \\ Drive, Ottawa ON K1A 0R6, Canada \\ ${ }^{2}$ Physics Department, Kansas State University, Manhattan, KS 66506, USA \\ ${ }^{3}$ National Research Council, 100 Sussex Drive, Ottawa ON K1A 0R6, Canada \\ E-mail: kirkwood@ieee.org, trallero@phys.ksu.edu and Paul.Corkum@nrc-cnrc.gc.ca
}

Received 18 April 2014, revised 15 July 2014

Accepted for publication 28 July 2014

Published 8 October 2014

\begin{abstract}
We demonstrated experimentally that the multiphoton ionization rate in gallium arsenide depends on the alignment of the laser polarization with respect to the crystal axis. We observed modulation in the ionization rate of a linearly-polarized $1900 \mathrm{~nm}$ laser beam directly by measuring its transmission while rotating the crystal, without Fourier analysis. We propose that the modulation in the ionization rate arises from periodic variation in the reduced carrier mass, as predicted by Keldysh theory. We show direct comparison of the experimental transmission modulation depth with Keldysh's non-resonant ionization theory for solids. This opens up a novel method for non-invasive crystallography of semiconductor materials.
\end{abstract}

Keywords: strong fields, ultrafast, ionization, GaAs, semiconductors

(Some figures may appear in colour only in the online journal)

\section{Introduction}

Fifty years ago, Keldysh published his theory on multiphoton ionization (MPI) that laid the foundation for strong field physics [1]. His atomic theory of ionization has been extensively studied [2] and refined [3, 4], leading to significant advances in our understanding of modern strong field phenomena in atomic and molecular systems such as attosecond pulse generation [5-8], high harmonic generation [9-13] and 'scanning tunnelling microscopy' of orbital structures [14].

Keldysh treated atoms and solids in a similar manner. Considering 'ionization' in a solid as the transition of an electron from the valence band to the conduction band, we can see the direct analogy between an atomic orbital and the band structure of a solid. By defining what we now call the 'Keldysh parameter', $\gamma$, MPI in these two systems were delineated between a tunnelling limit where $\gamma<1$, and a perturbative limit when $\gamma>1$. Much of modern strong field physics in atoms and molecules occurs on this dividing line where $\gamma \approx 1$.

Despite the advancements in our understanding of atomic and molecular multiphoton processes over these past decades, a similar revolution in our understanding of this process in solids has not occurred. The challenge in studying this process in solids is largely due to difficulties in isolating it from the complexity of interactions between a strong field and a high density of atoms in a periodic structure. Immediate experimental differences also arise when moving from atoms to solids since the products of ionization, such as electrons, ions, or high-harmonic generation, remain confined within the bulk. Several groups have studied ultrafast dynamics in solids in the weak field limit [15-18], but very little theoretical and experimental work has been done with strong fields. Early work in the strong field regime has been done in dielectrics [19, 20].

We can exploit the significantly higher density of electrons in solids $\left(10^{22} \mathrm{~cm}^{-3}\right.$ versus $10^{10} \mathrm{~cm}^{-3}$ in gases) to develop experiments that measure changes in the laser beam itself; specifically, the fractional change in transmission of the laser propagating through the material. Depletion of the laser beam due to nonlinear absorption gives an indirect measure of the ionization probability. Similar experiments on MPI in solids measuring changes in transmission were performed with dielectrics by Gertsvolf et al [19]. In these experiments, 


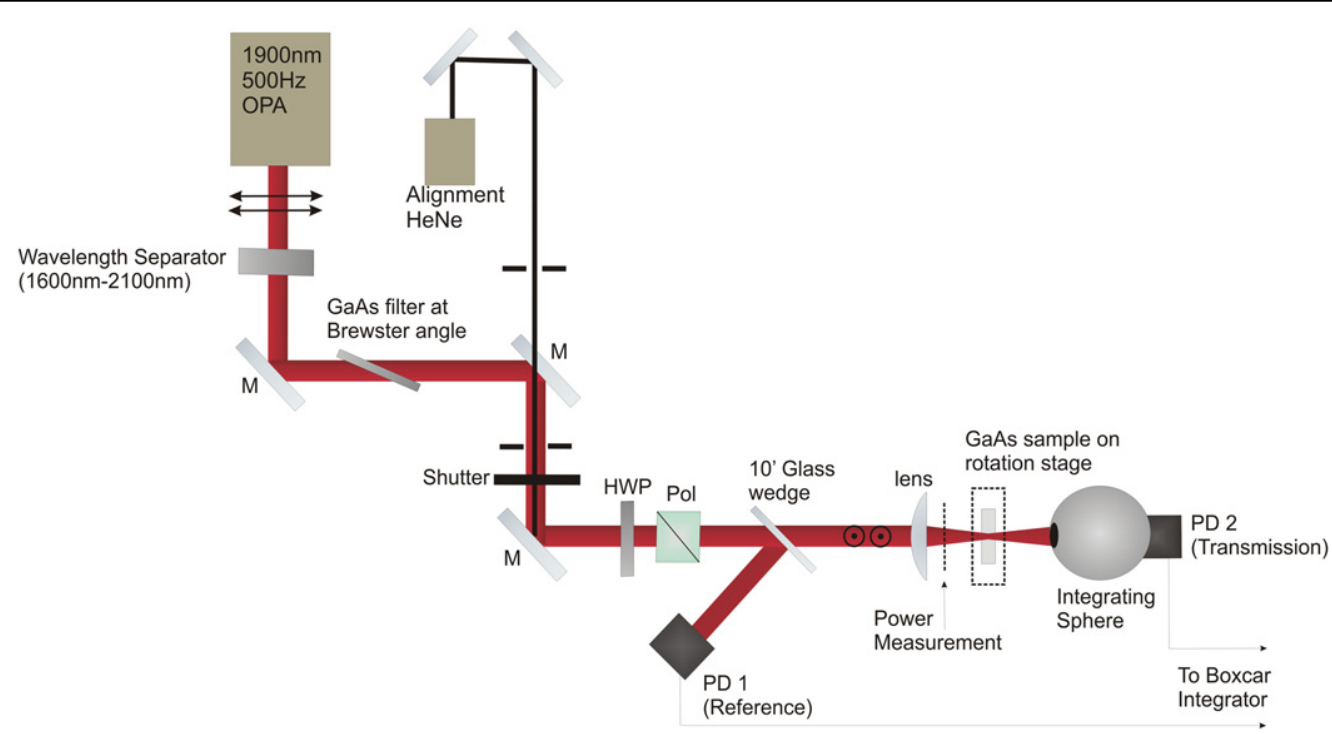

Figure 1. Experimental setup for measuring crystal structure with IR pulses from an OPA. The labels correspond to: HWP, half-wave plate; PD, photodiode; Pol, polarizer; M, mirror.

large bandgap crystals were ionized with a linearly-polarized, $800 \mathrm{~nm}$ laser beam and a periodic variation in the transmission was observed as a function of crystal rotation. The observed periodicity matched the symmetry of the crystal, which was linked to differences in the MPI rate.

Keldysh's theory on MPI in solids is dependent on the effective carrier mass, directly related to band curvature near the minimum bandgap. The theory assumed a parabolic band structure, requiring a constant and definitive effective mass. Despite the more complicated band structures in the dielectrics tested, those experiments showed promising qualitative results. Moreover, it has been recently shown that light-induced currents in dielectrics can be created with strong fields [20]. With the observation of similar atomic strong field processes in semiconductors, such as high harmonic generation [21, 22], interest has increased in Keldysh's treatment of solids. The recent developments in longer wavelength femtosecond lasers into the mid-IR [23] now allow us to study semiconductors where new photonic devices can be developed. These lasers also allow us to isolate this ionization process from linear, resonantly nonlinear and thermal processes, and test for more accurate theoretical predictions, as was done with atoms and molecules these past decades.

In this manuscript, we show that strong-field ionization of solids near the tunnelling limit can be used to trace the crystal symmetry and reveal interband absorption dynamics near the bandgap of solid periodic structures. By rotating a GaAs crystal about a linearly-polarized, infrared femtosecond laser beam, we measured periodic variations in the fractional transmission and compared it to predictions based on Keldysh's MPI model for solids. Our comparisons are further aided by more accurate predictions of the effective mass through simulations using density functional theory (DFT).

\section{Experimental setup}

To measure the crystal structure of GaAs using Keldysh's theory for solids, we selected the idler output from a superfluorescence-seeded fs-TOPAS optical parametric amplifier (OPA) made by Light Conversion, which was pumped by a regeneratively-amplified titanium-doped sapphire (Ti:S) laser. The Ti:S was a homemade system having a center wavelength of $800 \mathrm{~nm}$, which produced $50 \mathrm{fs}$ full-width at half-maximum (FWHM) pulses at a repetition rate of $500 \mathrm{~Hz}$ with a maximum pulse power of $800 \mu \mathrm{J}$. The linearly-polarized OPA idler beam was adjusted to produce $1900 \mathrm{~nm}(0.6 \mathrm{eV}), 75 \mathrm{fs}$ (FWHM) pulses.

A schematic of our experimental setup is shown in figure 1. The OPA output was filtered by a set of dielectric mirrors to select the idler beam followed by a $600 \mu \mathrm{m}$ thick GaAs crystal set at the Brewster angle $\left(73^{\circ}\right)$ to filter out any residual wavelengths created through additional nonlinear processes within the OPA.

The idler beam was passed through an electronicallycontrolled shutter to control the exposure time for each crystal angle. A half-wave plate and polarizer were used to vary the incident pulse energy, and the angle of the half-wave plate was also automated to control the input energy of the idler beam. A photodiode (PD1) was calibrated against the incident pulse energy, measured by a power meter placed at a reference plane between the lens and the sample to account for Fresnel losses from the lens surfaces, as illustrated by the dotted line in figure 1.

In gas ionization theory, the ionization threshold approximately scales with the ionization potential as $I_{\text {th }} \propto I_{\mathrm{P}}^{4}$, independent of the wavelength. Treating the bandgap in a solid as analogous to the ionization potential, we estimated a minimum intensity above which to measure ionization. In dielectrics with a bandgap of $9 \mathrm{eV}$, the ionization threshold was approximately $10^{13} \mathrm{~W} \mathrm{~cm}^{-2}$. Scaling down to the $1.43 \mathrm{eV}$ 

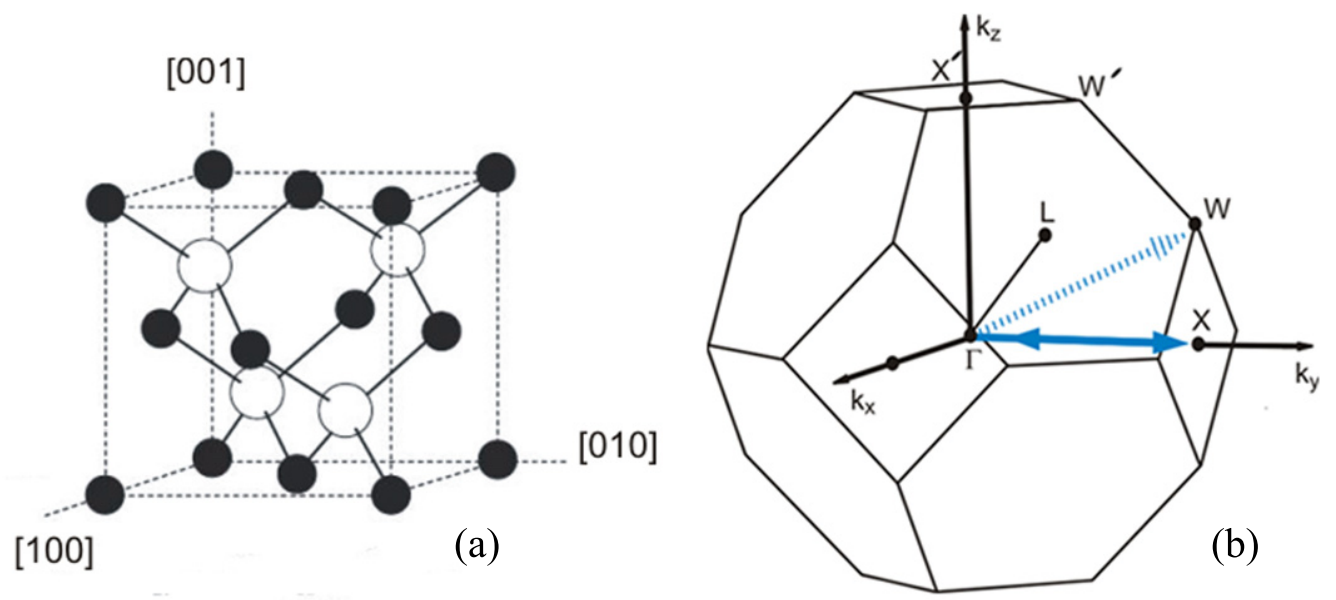

Figure 2. (a) FCC cubic structure of GaAs as viewed along the [100] axis. The black and white spheres represent the gallium and arsenic atoms, respectively; (b) GaAs reciprocal lattice (first Brillouin zone). The propagation axes are marked as $k_{i}$. The high symmetry points are labelled $\Gamma, X, W, L$.

bandgap of GaAs, an ionization threshold of approximately $10^{10} \mathrm{~W} \mathrm{~cm}^{-2}$ was predicted. Our pulse energy on the target could vary from $0.9 \mu \mathrm{J}$ to $6 \mu \mathrm{J}$, with a $1.1 \mathrm{~mm}$ incident beam radius (1/e) as measured using a knife-edge, our intensity range varied from $3 \times 10^{10} \mathrm{~W} \mathrm{~cm}^{-2}$ to $2 \times 10^{11} \mathrm{~W} \mathrm{~cm}^{-2}$ when focussed with a $200 \mathrm{~mm}$ focal length plano-convex lens.

The sample was mounted on a rotation stage which itself was mounted on a two-dimensional translation stage to allow us to select any spot on the surface. In addition, a kinematic mount was attached to the rotation stage to align the sample for normal incidence. The laser transmission through the sample was collected with an integrating sphere to ensure the entire beam was collected. Traces from both the transmission and reference photodiodes were processed with a boxcar integrator and sent to a data acquisition card. This setup allowed for single shot measurements synchronized to the laser shutter.

\section{Crystal properties}

At a wavelength of $1900 \mathrm{~nm}$, the semi-insulating GaAs(100) with a bandgap of $1.43 \mathrm{eV}$ was transparent, giving negligible linear absorption. GaAs is not birefringent when light propagates along a principal axis and should not induce any additional symmetry dependence. Since these experiments involved at least three-photon absorption, we can disregard all two-photon absorption effects. Finally, because of the high electron carrier mobility, the electrons quickly replenish between laser pulses.

GaAs has a face-centred cubic (FCC) zinc-blende structure as shown in figure 2. The structure space group is $F \overline{4} 3 \mathrm{~m}$. Since it is a cubic structure, it has a four-fold symmetry and the angles of the unit cell are all at $90^{\circ}$. Figure 2 is viewed along the (100) plane, which can be seen as parallel to the $i-j$ axes and orthogonal to the $k$ axis. The (100) plane is identical to the (010) and (001) planes.

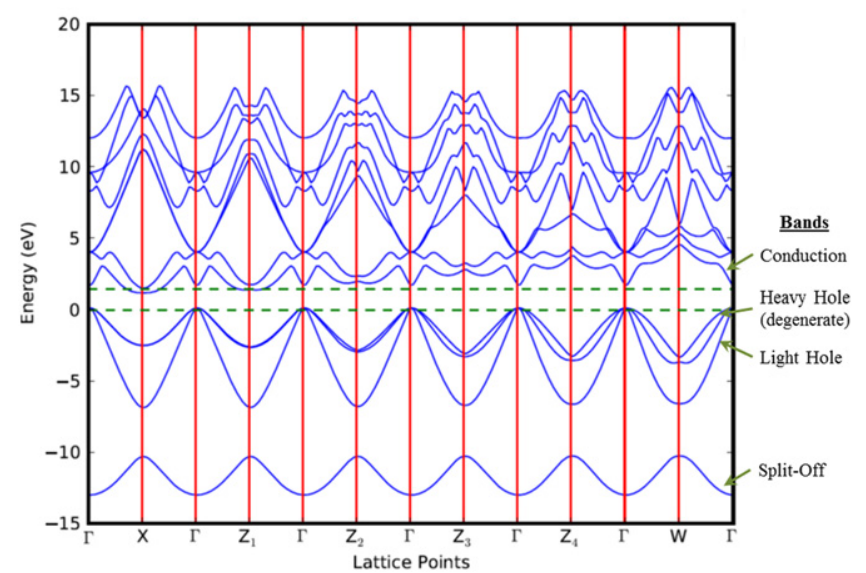

Figure 3. Band structure for increasing sample angle about the (100) propagation axis, from $X$ to $W$, as calculated using DFT. Green dotted lines highlight the bandgap.

The first Brillouin zone is shown in figure 2. The $\Gamma$ point is at the origin with zero momentum and the boundary sits at a momentum of $k_{i}=2 \pi / a$. The high symmetry points $(\Gamma, X$, $W, L)$ can be visualized more clearly in reciprocal space and all lie on the boundary. As expected, the GaAs reciprocal lattice has a four-fold symmetry, analogous to the GaAs realspace lattice. Due to the direct relationship between the spaces, the laser can be visualized as propagating along direction, $k_{i}$, where $i$ can be any of $x, y$, or $z$.

For our experiments, we need to know the valence and conduction band curvatures along the laser polarization direction as the crystal is rotated about the propagation axis. We modeled the GaAs band structure around the axis of rotation using ABINIT [24], a DFT with pseudopotential functions. Figure 3 shows the band structure for the first section of the Brillouin zone (from $X$ to $W$ ). The band structure can be envisioned along paths directing in and out from the central point, $\Gamma$; first to point $X$ and then to incrementing points, $Z_{i}$ in increasing angles about the propagating axis, $k_{x}$. Points $Z_{i}$ are initially defined in reciprocal space and 


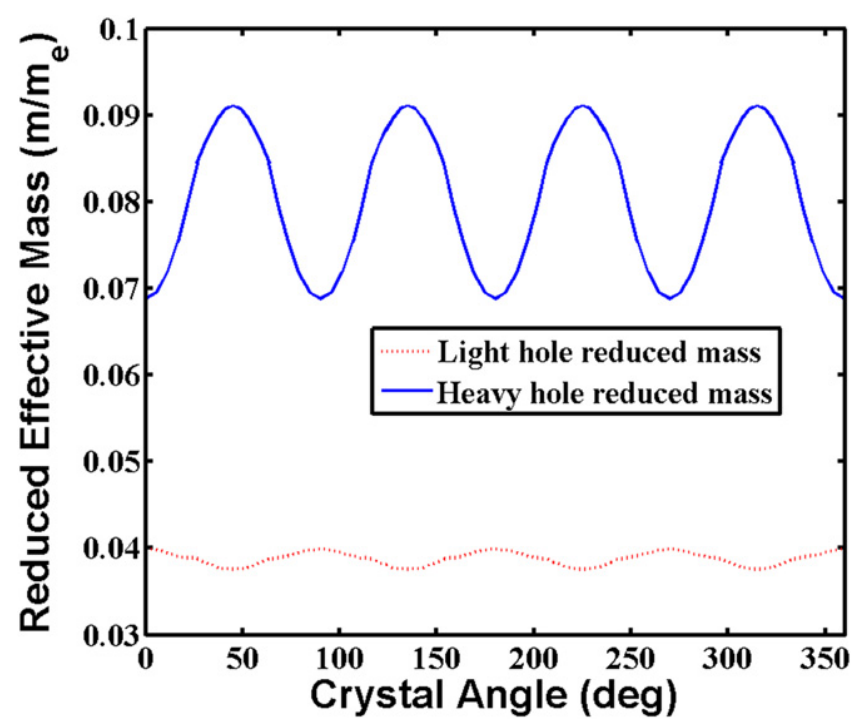

Figure 4. Reduced mass of GaAs calculated as a function of the GaAs crystal angle, using the second derivative of the DFT bandstructure. The blue curve represents a heavy-hole valence band transition to the conduction band and the red curve represents a lighthole transition.

then converted into real space to find the corresponding realspace angle. Similar curves were made for the rotation from $W$ to $W^{\prime}$, and $W^{\prime}$ to $X^{\prime}$. Due to the four-fold symmetry, the pattern repeats in each quadrant.

The effective mass of a carrier in a periodic potential is dependent on the curvature of the band as shown in the following equation [25]:

$$
m^{*}=\hbar^{2}\left(\frac{\mathrm{d}^{2} \varepsilon}{\mathrm{d} k^{2}}\right)^{-1},
$$

where $\varepsilon$ is the band energy and $k$ is the lattice vector. The second derivative of the band structure at the $\Gamma$ point for each angle was calculated numerically from the DFT data using a least-squares fitting algorithm, the Savitzky-Golay method [26].

Since ionization involves the transition of a carrier from one energy surface to the next, the overall mass of the carrier being ionized is the reduced mass, defined as,

$$
\frac{1}{m^{*}}=\frac{1}{\left|m_{\mathrm{c}}^{*}\right|}+\frac{1}{\left|m_{\mathrm{v}}^{*}\right|},
$$

where $m_{v}^{*}$, and $m_{c}^{*}$ are the effective masses of the valence band and conduction band, respectively. The absolute values were used since the sign of the mass infers directionality of the carrier acceleration.

The reduced mass for ionization from either the heavy hole or light hole valence band is shown in figure 4 . The light hole reduced mass varies with a smaller amplitude than the heavy hole mass and the phase of the two curves differ by $90^{\circ}$. As the amount of laser transmission is expected to be inversely related to the reduced mass [19], for GaAs the transmission modulation phase will provide further evidence from which band the carrier ionized. For example, when

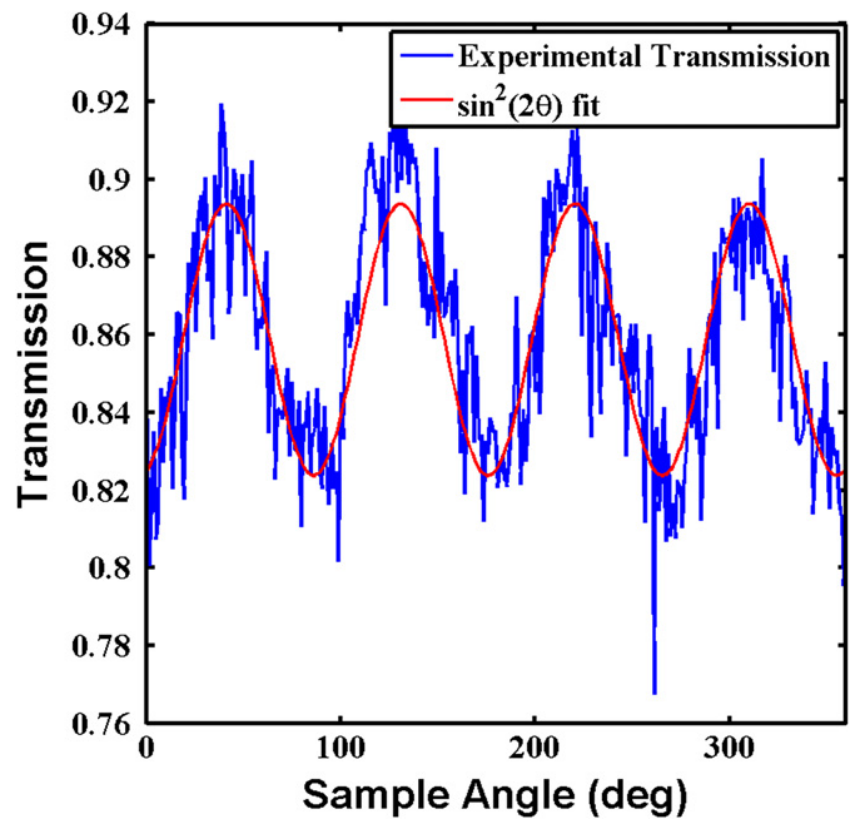

Figure 5. Modulation of laser transmission through $600 \mu \mathrm{m} \mathrm{GaAs}$ (100) crystal with a linearly-polarized $1900 \mathrm{~nm}$ laser beam having an incident energy of $6 \mu \mathrm{J}$. The $0^{\circ}$ corresponds to the [001] axis. The experimental data is fit to a $\sin ^{2}(2 \theta)$ fitting curve and shown as the smooth red curve.

comparing transmission from $0^{\circ}$ as compared to $45^{\circ}$, one would expect a transition from a maximum to a minimum if ionization dominantly occurred from the heavy hole valence band, with the opposite occurring if the light hole valence band dominated.

\section{Experimental results}

When the sample was rotated in a plane normal to the laser propagation direction, the transmission was measured for each angle, $\theta$ as shown in figure 5. The angle, $\theta$, is defined as the sample's angle with respect to the polarization axis of our linearly-polarized laser beam, where $0^{\circ}$ corresponds to alignment of the [001] axis with the laser polarization.

The transmission, $T$, was defined as

$$
T=\frac{E_{\mathrm{out}}}{E_{\mathrm{in}}}=\frac{E_{\mathrm{in}}-E_{\mathrm{abs}}}{E_{\mathrm{in}}},
$$

where $E_{\mathrm{abs}}, E_{\mathrm{in}}$, and $E_{\text {out }}$ are the energy absorbed, input pulse energy, and output pulse energy, respectively.

In addition to transmission, we defined a modulation depth by first fitting a $A+B \sin ^{2}(2 \theta)$ curve to the experimental data using MATLAB's least square fit, where $A$ and $B$ are fitting parameters. The modulation depth was defined as the difference between the maximum and minimum transmission. These curves were reproduced for the laser energy range discussed in the experimental section.

The least square error between the modulation data and the $\sin ^{2}(2 \theta)$ fit is approximately $2 \%$, which is similar to the pulse-to-pulse fluctuations of the laser. From this fitting 


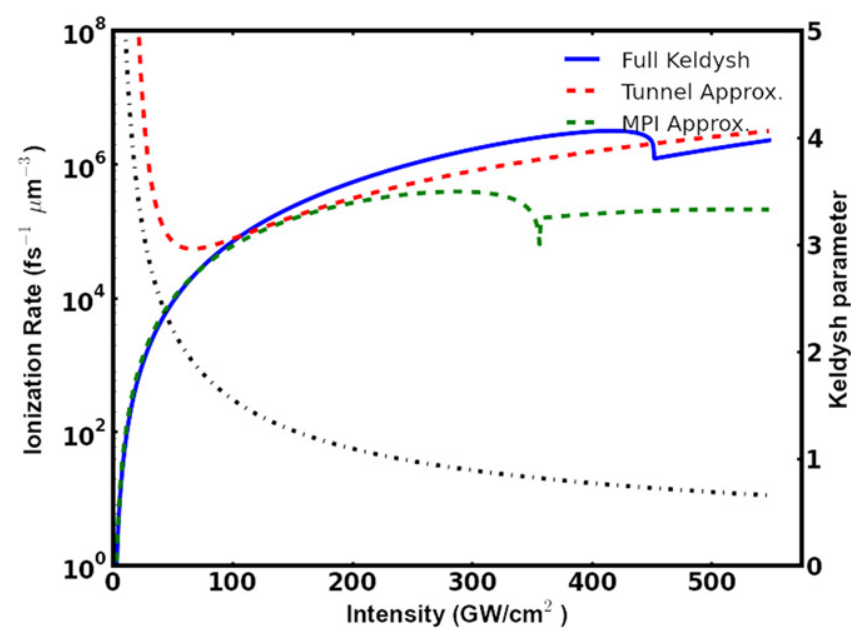

Figure 6. Ionization rate in $\mathrm{GaAs}(100)$ using the Keldysh approximation of tunnelling and multiphoton approximations, in red and green, respectively. The full Keldysh ionization model is shown with the solid blue line. The ionization rates were modeled with a $1900 \mathrm{~nm}$ laser and an effective mass of $0.06877 \mathrm{~m}_{\mathrm{e}}$. The black dash-dot line is the Keldysh parameter plotted on the right.

curve, we find the period to be $\sim 90^{\circ}$, which confirms the fourfold symmetry expected for the cubic GaAs crystal.

In figure 5 , we see that the minimum transmission (maximum absorption) occurs at the $0^{\circ}$ angle. This alignment was evident as the crystal would cleave parallel and perpendicular to the wafer notch. Due to symmetry, we get the same transmission every $90^{\circ}$, which corresponds to the [010], [00 $\overline{1}$ ], and [010] axes for $\theta=90^{\circ}, 180^{\circ}$, and $270^{\circ}$, respectively. The sample was rotated over several revolutions and showed no change in modulation, thus revealing no evidence of sample damage. Recall from figure 4 in the previous section that a minimum transmission at $0^{\circ}$ would be the signature of a heavy hole rather than a light hole valence band transition.

\section{Theory}

To use our experimental results to test Keldysh's theory of ionization in solids using the reduced carrier masses from DFT, as discussed earlier, we need to calculate the transmission of a laser pulse through a solid ionized in this manner.

The Keldysh model divides MPI into two regimes: perturbative MPI and tunnelling ionization. In the MPI regime, the electron absorbs multiple laser photons to excite an electron from the valence band to the conduction band. In the tunnelling regime, the energy bands are suppressed by the strong electric field, allowing the electron to tunnel from the valence band, through the potential barrier and into the conduction band before the electric field reverses sign. Tunnelling only occurs if the laser frequency is slower than the mean-free transit time of the electrons. The Keldysh parameter for solids, which delinates between these regimes, was defined as [1]

$$
\gamma=\omega \frac{\sqrt{E_{g} m^{*}}}{e F},
$$

where $\omega, E_{g}, m^{*}$, and $F$ are the angular frequency, bandgap energy, effective mass, and field strength, respectively. This Keldysh parameter for solids differs from the atomic case by a factor of $1 / \sqrt{2}$.

In the tunnelling regime, the transition rate can be written in terms of $\gamma$ as,

$$
\begin{aligned}
w_{\text {Tunnel }}(\gamma)= & \frac{2}{9 \pi} \frac{E_{\mathrm{g}}}{\hbar}\left(\frac{m^{*} E_{\mathrm{g}}}{\hbar^{2}}\right)^{3 / 2}\left(\frac{\hbar \omega}{E_{\mathrm{g}}} \frac{1}{\gamma}\right)^{5 / 2} \\
& \times \exp \left\{-\frac{\pi}{2} \frac{E_{\mathrm{g}}}{\hbar \omega} \gamma\left(1-\frac{1}{8} \gamma^{2}\right)\right\} .
\end{aligned}
$$

From this equation, we see that the tunnelling regime begins to break down when $\frac{1}{8} \gamma^{2}>1$ or $\gamma>2.8$ (as the intensity decreases). As $\gamma \gg 1$, the rate enters the perturbative multiphoton regime (MPI) and the expression becomes,

$$
\begin{aligned}
w_{\text {MPI }}(\gamma)= & \frac{2}{9 \pi} \omega\left(\frac{\omega m^{*}}{\hbar}\right)^{3 / 2} \Phi\left((2\langle x+1\rangle-2 x)^{1 / 2}\right) \\
& \times \exp \left\{2\langle x+1\rangle\left(1-\frac{1}{4 \gamma^{2}}\right)\right\}\left(\frac{1}{16 \gamma^{2}}\right)^{\langle x+1\rangle},
\end{aligned}
$$

where $x=\tilde{\Delta} / \hbar \omega, \Phi$ is the Dawson integral, the brackets \langle\rangle represents the integer portion, and $\tilde{\Delta}$, is the effective bandgap energy. In the MPI regime, the effective bandgap can be written as,

$$
\tilde{\Delta}=E_{g}+\frac{e^{2} F^{2}}{4 m^{*} \omega^{2}},
$$

where the second term in this expression is equivalent to the ponderomotive energy.

In these sets of experiments, the intensity range spans both the MPI and tunnelling regimes necessitating the use of the full theory. Using the following simplifying expressions:

$$
\begin{aligned}
\gamma_{\mathrm{f}} & =\frac{1}{\sqrt{1+\gamma^{2}}}, \\
E_{1} & =E\left(\gamma_{\mathrm{f}}\right) ; E_{\gamma}=E\left(\gamma \gamma_{\mathrm{f}}\right) ; K_{1}=K\left(\gamma_{\mathrm{f}}\right) ; \\
K_{\gamma} & =K\left(\gamma \gamma_{\mathrm{f}}\right),
\end{aligned}
$$

where $E(\gamma)$ and $K(\gamma)$ are elliptic integrals of the first and second kind, and generalizing the effective bandgap for all values of $\gamma$ as

$$
\tilde{\Delta}=\frac{2 E_{1}}{\pi \gamma \gamma_{f}} E_{g}
$$

which recovers the MPI limit in equation (7), we can rewrite 
the Keldysh model for solids, equation (37) in [1], as

$$
\begin{aligned}
w(\gamma)= & \frac{2 \omega}{9 \pi}\left(\frac{m^{*} \omega}{\hbar \gamma \gamma_{f}}\right)^{\frac{3}{2}} Q\left(\gamma, \frac{\tilde{\Delta}}{m^{*} \omega}\right) \\
& \times \exp \left\{-\pi\left\langle\frac{\tilde{\Delta}}{\hbar \omega}+1\right\rangle\right\} \frac{K_{\gamma}-E_{\gamma}}{E_{1}},
\end{aligned}
$$

where the function $Q$ is

$$
\begin{aligned}
Q(\gamma, x)= & \sqrt{\frac{\pi}{2 K_{1}}} \sum_{n=0}^{\infty} \exp \left\{-\pi n \frac{K_{\gamma}-E_{\gamma}}{E_{1}}\right\} \\
& \times \Phi\left(\sqrt{\frac{\pi^{2}(2\langle x+1\rangle-2 x+n)}{2 K_{1} E_{1}}}\right)
\end{aligned}
$$

and the index, $n$, indicates the multiphoton order. Note that the effective bandgap $\tilde{\Delta}$ increases with increasing laser intensity, which we refer to as channel closing as the intensity of the laser increases.

Figure 6 shows the ionization rate for GaAs at $1900 \mathrm{~nm}$ extending a bit beyond our intensity range using a reduced mass of $0.06877 \mathrm{~m}_{\mathrm{e}}$ (heavy hole transition at $0^{\circ}$ ). The figure shows that ionization is effectively modeled using the perturbative MPI approximation at low intensities (high $\gamma$ ), equation (6). As $\gamma$ drops below 2.8, with increasing intensity, we approach the tunnelling regime and the tunnelling approximation in equation (5) is valid. The full Keldysh MPI model in solids, equation (10), shows a sharp dip related to channel closing as the effective bandgap increases and four photons are required to span the gap rather than three. The tunnelling approximation does not include this effect, and although the perturbative approximation includes channel closing, it models it at a lower intensity with an overall lower ionization rate, which justifies using the full model in this transition region between approximations where $\gamma \approx 1$.

\section{Discussion}

In figure 7 , the transmission of the $1900 \mathrm{~nm}$ laser beam for a fixed incident polarization angle of $0^{\circ}$ was plotted normalized to the transmission of a linearly-absorbed laser beam (accounting for Fresnel losses). The curves in figure 7 were calculated by propagating an initially Gaussian temporal and spatial intensity distribution through a $600 \mu \mathrm{m}$ thick sample and depleting the simulated pulse using the space-time averaged Keldysh ionization rate with the reduced mass between the lowest conduction band and the heavy hole valence band, for the blue curve, and the light hole valence band for the dashed red curve.

The energy absorbed by the material in each time step, $\mathrm{d} E_{\mathrm{abs}}$, is given below,

$$
\mathrm{d} E_{\mathrm{abs}}=E_{g} w(I) N \mathrm{~d} t
$$

with $w(I)$ being the space-time averaged Keldysh ionization rate for a given reduced mass, and $N$ being the electron number density.

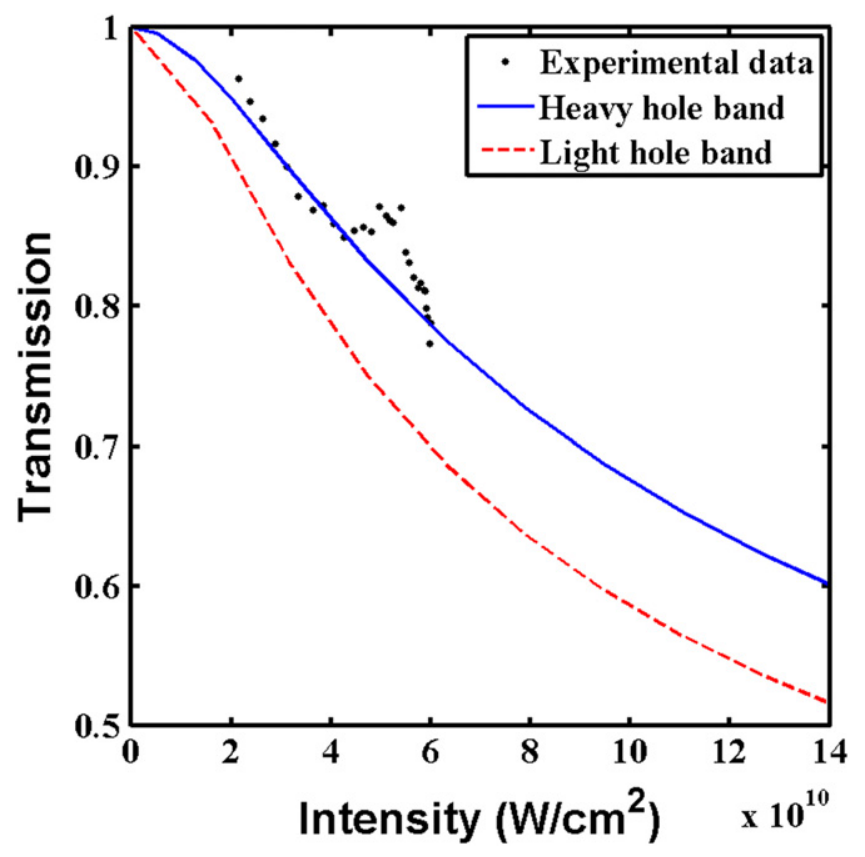

Figure 7. Transmission through $600 \mu \mathrm{m} \mathrm{GaAs}(100)$ with a $1900 \mathrm{~nm}$ laser beam at an angle of $0^{\circ}$. The data markers represent the experimental data, and the solid blue and dashed red lines are the theoretical transmission curves for the heavy and light hole band ionization, respectively.

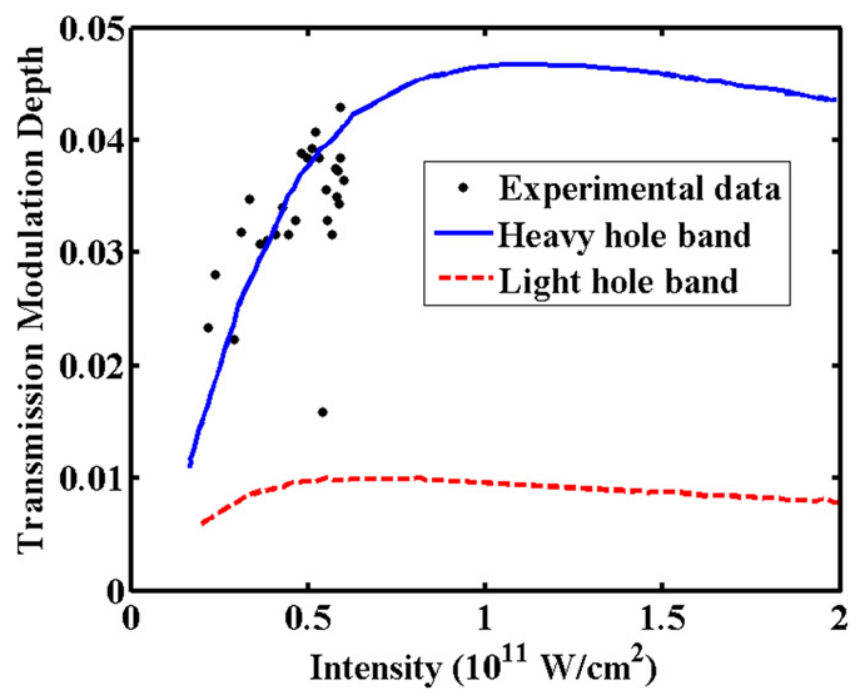

Figure 8. Modulation depth for transmission of $1900 \mathrm{~nm}, 75 \mathrm{fs}$ pulse through a $600 \mu \mathrm{m}(100) \mathrm{GaAs}$, as a function of changing intensity. The points represent the experimental data and the solid blue and dashed red lines are the Keldysh transmission curves for the heavy and light hole band ionization respectively.

Although the independent laser parameters: pulse energy, pulse width, and incident beam waist, were measured and should lead to an accurate calculation of the incident intensity, the experimental and the theoretical transmission data were still offset in intensity. We measured the pulse width of the $800 \mathrm{~nm}$ laser to be $50 \mathrm{fs}$ (FWHM), but a corresponding measurement of the idler pulse width was not possible. We estimated the pulse would stretch to about $75 \mathrm{fs}$ from $50 \mathrm{fs}$ based on similar OPA pulse measurements. Furthermore, the 
knife-edge measurement of the incident beam indicated additional structure to the beam, possibly increasing the $M^{2}$ above one, leading to a larger focal spot. For these initial experiments, we did not attempt to clean the spatial mode.

In gas ionization and high-harmonic generation, similar variabilities in the prediction of the laser intensity from the independent measurements and the focal spot conditions have been observed [27, 28]. As in those experiments, we used the transmission curve to assess the experimental intensity of the laser at focus inside the sample, similar to how the ionization threshold calibration was used in gas-phase strong field experiments. Energy loss due to Fresnel reflection off the front and back sample surfaces was corrected using the reflection coefficient of GaAs at $1900 \mathrm{~nm}$. Since the phase of the modulation curve seen in figure 5 confirms that the heavy hole valence electrons are predominantly ionized, fitting the experimental data to the heavy hole curve in figure 7 is further justified.

The transmission decreases as the laser pulse intensity increases because the beam is depleted by absorption of the laser energy required for carrier band-to-band transitions. The steep decline in transmission as a function of intensity shows evidence of nonlinear absorption, since linear absorption would only depend on the sample thickness and not the pulse energy. The hump seen in the experimental data at $\sim 5 \times 10^{10}$ $\mathrm{W} \mathrm{cm}{ }^{-2}$ is not repeatable and thus part of the experimental error. These experiments were repeated and this current data set was chosen for completeness.

Figure 8 shows the modulation depth as a function of intensity inside the sample using the intensity as calibrated by the transmission curve of figure 7 . The points represent the experimental data and the solid blue and dashed red lines are the theoretical transmission curves for the heavy and light hole band ionization, respectively. The large fluctuation seen in the experiment around $5 \times 10^{10} \mathrm{~W} \mathrm{~cm}^{-2}$ is due mainly to power fluctuations from the laser propagating through the OPA.

The theoretical curves show an initial increase in modulation depth as the ionization rate is angle-dependent. Figure 5 shows that there is little ionization at $(2 n+1) \pi / 4$ degrees compared to ionization at $n \pi / 2$ degrees, where $n \in \mathbb{Z}$. As the intensity continues to increase, the modulation depth plateaus when ionization at $(2 n+1) \pi / 4$ degrees is no longer negligible. At much larger intensities, the modulation depth decreases as the pulse is depleted of photons, showing less variation between transmission along different sample angles.

Given the high intensity and short pulse width of the laser pulse, other nonlinear effects could result in transmission variations as explored here, in particular self-focusing and phase advance. In the case of self-focusing, the beam would experience an increase in the refractive index from the intensity-dependent refractive index. For GaAs, the nonlinear index is $n_{2}=3.3 \times 10^{-13} \mathrm{~cm}^{2} \mathrm{~W}^{-1}$. Self-focusing would overcome the beam's divergence if its power was above a critical power, [29], which is $4.8 \mathrm{~kW}$ for $1900 \mathrm{~nm}$ propagating through GaAs(100) crystal.
Rather than stay below the critical power, we used a relatively large beam size so that the change in beam waist due to self-focusing would be negligible. We chose a focusing lens of $200 \mathrm{~mm}$, which gives a predicted beam waist of 110 $\mu \mathrm{m}$ and a peak power greater than $1 \mathrm{MW}$. The Rayleigh range was $2 \mathrm{~cm}$, which is over 30 times the thickness of the sample.

Using the maximum energy output of the $1900 \mathrm{~nm}$ laser system $(\sim 40 \mu \mathrm{J})$, the distance over which the beam would collapse due to self-focussing was calculated to be $1.4 \mathrm{~mm}$ [29]. Using simple geometry, the output intensity would increase by a factor of 1.6, purely due to self-focusing.

As shown in [30], however, ionization occurs at the peak of the laser as it approaches the focal spot, depleting and clamping the peak intensity. Using equation (10) and the ionization simulations described previously, we found that the intensity falls to approximately $1 / e$ times its maximum value upon exiting the sample, limiting the effect of self-focusing.

Phase advance may occur when the refractive index is decreased due to plasma generation in the material, causing the beam to diverge (or self-defocus). As the material is ionized by an incoming electric field, the carrier density (plasma) will increase, reducing the refractive index. We used the classical electron oscillator model (Drude model for dielectrics and semiconductors) to find the change in index to be,

$$
\Delta n(\lambda)=-\frac{e^{2} \lambda^{2} \Delta N}{8 \pi^{2} n_{0} \epsilon_{0} c^{2} m},
$$

where $N$ is the free electron (plasma) density in $\mathrm{cm}^{-3}$, and $m$ is the effective mass, and $n_{0}$ is the refractive index.

We estimated that the minimum carrier density needed to achieve a phase advance of $\pi$ to be $9.66 \times 10^{15} \mathrm{~cm}^{-3}$ for $1900 \mathrm{~nm}$. For an input Gaussian beam, the input laser fluence is given by $\sim I \tau_{\mathrm{FWHM}}$. The fluence removed from the beam through ionization to reach a phase advance of $\pi$ is $\sim N_{\pi} E_{g} L$, assuming a constant interaction region over the full length of the sample. We estimated the minimum transmission for which the phase advance becomes significant by taking the ratio of remaining fluence in the beam over the input laser fluence. We found that for $1900 \mathrm{~nm}$ pulses, the minimum transmission is 0.56 . Using our Keldysh model, we find that this transmission value is not reached until the peak intensity exceeds $14 \times 10^{10} \mathrm{~W} \mathrm{~cm}^{-2}$, which was not possible in our experiments. The lowest transmission achieved in the $1900 \mathrm{~nm}$ experiments was $\sim 0.75$ meaning that phase advance was negligible.

The experimental data again shows that Keldysh ionization from the GaAs heavy hole valence band predicts the ionization rate. Although the ionization rate of the light hole valence band is greater than that for the heavy holes, the heavy hole valence band shows a much greater variation in reduced mass as a function of angle. The ionization rate from the heavy hole band varies by $33.7 \%$ while the light hole band varies by only $8.7 \%$ (Both depths are calculated theoretically for a peak intensity of $10 \times 10^{10} \mathrm{~W} \mathrm{~cm}^{-2}$ and averaged over the laser spot). 
The transmission experiments used to measure crystal symmetry in dielectrics by Gertsvolf et al [19] also used the Keldysh ionization rate. Using DFT to produce the band strucutre for various dielectrics, they showed that the reduced mass was angle-dependent when using a linearly polarized, $800 \mathrm{~nm}$ laser beam on a rotated sample. With parabolic fitting at the band minimum for direct bandgap dielectrics, the ionization was shown to be most probable between the top of the valence band to the bottom of the conduction band, as shown here, due to the exponential-dependence on the bandgap. In that work, they discovered that the difference in the reduced mass between two symmetry points, a factor of 2 in quartz, was not a predictor in the difference in the ionization rates. They measured a difference of about $1 \%$ and required Fourier analysis to visualize the crystal symmetry. As seen in figure 5, the modulation depth for GaAs varied from $2 \%$ to $5 \%$, which allowed us to reveal the crystal symmetry directly without the need of Fourier analysis. This difference in modulation depth for GaAs results from a much smaller variation in our reduced masses between our symmetry points than seen in dielectrics.

Further evidence of ionization from the heavy hole valence band results from calculating the density of states for each band from our DFT results. In the $\Gamma-\mathrm{X}$ to $\Gamma-\mathrm{W}$ directions, the conduction band DOS decreases from $2 \times 10^{17}$ $\mathrm{cm}^{-3}$ to approximately $1.5 \times 10^{17} \mathrm{~cm}^{-3}$ at $\mathrm{W}$. As for the heavy hole valence band, there is a degeneracy of 2 and the band responsible for modulation used in this work has a DOS that increases from $4.7 \times 10^{17} \mathrm{~cm}^{-3}$ to $8.8 \times 10^{17} \mathrm{~cm}^{-3}$. The light hole valence band decreases from $1.1 \times 10^{17} \mathrm{~cm}^{-3}$ to $0.7 \times 10^{17} \mathrm{~cm}^{-3}$. Although the ionization rate from the light hole valence band should be higher given the smaller reduced mass, the conduction band would not be filled by the ionized light valence electrons and the higher density of heavy hole valence electrons would dominate ionization.

Also observed in Gertsvolf et al [19], transmission variations with crystal symmetry in quartz vanished when the photon energy was doubled using $400 \mathrm{~nm}$ laser pulses. The absence of this variation was attributed to the ionization transitioning into the perturbative regime, where $\gamma>1$. The variation in transmission was still observed in $\mathrm{LiF}$, though, and this variation was attributed to the ionization still being in the tunnelling regime with four-photon absorption. In our work, we are not in a purely tunnelling regime as shown plotted to the right in figure 6 and ionization only required three photons for absorption as the effective bandgap would not exceed this energy until the intensity increased beyond $40 \times 10^{10} \mathrm{~W} \mathrm{~cm}^{-2}$. Although we were in a three-photon absorption regime where ionization could not be said to purely tunnelling, a significant variation in transmission was observed counter to that in observed in quartz at $400 \mathrm{~nm}$ by Gertsvolf et al. The complexity of the band structure in quartz, as compared to $\mathrm{LiF}$ and GaAs here, may be a factor, providing more incentive to explore Keldysh's ionization theory in solids with different crystal structures, especially as longer wavelength lasers become available.

\section{Conclusion}

We demonstrated that our experimental technique confirms theoretical predictions of MPI inside bulk GaAs using Keldysh's non-resonant MPI theory for solids. This evidence was revealed as a decrease in the transmission of $1900 \mathrm{~nm}$ pulses as a function of laser intensity. In addition, we experimentally show that the MPI (band-to-band excitation) of GaAs(100) reveals the crystal's symmetry. This symmetry was observed by rotating the laser polarization with respect to the crystal axis and measuring the laser transmission. We observed a distinct modulation in the transmission that repeated every $90^{\circ}$, congruous to the four-fold symmetry of a GaAs cubic fcc crystal. Prediction of semiconductor crystal symmetry followed similar results seen by Gertsvolf et al [19] with dielectrics. The modulation depth was more significant than seen in any previous work, allowing visualization of crystal symmetry without further Fourier analysis.

The experimentally measured transmission and the modulation depth were predicted by Keldysh's non-resonant MPI theory for solids. In this theory, ionization depended on the reduced mass, which results from the curvature of the band structure along different directions of laser polarization. We showed that the modulated transmission results primarily from ionization between the heavy hole valence band and the conduction band at the direct bandgap.

Fifty years after the publication of Keldysh's theory of MPI and the signficant advances made in his atomic theory for atoms and molecules in predicting modern strong field phenomena, these experiments and the development of longer mid-IR wavelength lasers will open the way to similar advances in strong field ionization in solids.

\section{Acknowledgement}

SMG was supported by an NSERC graduate student scholarship.

\section{References}

[1] Keldysh L V 1965 Ionization in field of a strong electromagnetic wave Sov. Phys.-JETP 201307

[2] Augst S, Meyerhofer D D, Strickland D and Chin S L 1991 Laser ionization of noble gases by Coulomb-barrier suppression J. Opt. Soc. Am. B 8 858-67

[3] Ammosov M V, Delone N B and Krainov V P 1986 Tunnel ionization of complex atoms and of atomic ions in an alternating electromagnetic field Sov. Phys._JETP 641191

[4] Perelomov A M, Popov V S and Terent'ev M V 1966 Ionization of atoms in an alternating electric field Sov. Phys. -JETP 23

[5] Krausz F and Yu Ivanov M 2009 Attosecond physics Rev. Mod. Phys. 81 163-234

[6] Chang Z 2011 Fundamentals of Attosecond Optics (Boca Raton, FL: CRC Press)

[7] Corkum P B and Krausz F 2007 Attosecond science Nat. Phys. 3 381-7 
[8] Corkum P B and Chang Z 2008 The attosecond revolution Opt. Photonics News 19 24-29

[9] Ferray M, L'Huillier A, Li X F, Lompre L A, Mainfray G and Manus C 1988 Multiple-harmonic conversion of $1064 \mathrm{~nm}$ radiation in rare gases J. Phys. B: At. Mol. Opt. Phys. 21 L31

[10] Macklin J J, Kmetec J D and Gordon C L 1993 High-order harmonic generation using intense femtosecond pulses Phys. Rev. Lett. 70 766-9

[11] L'Huillier A and Balcou Ph 1993 High-order harmonic generation in rare gases with a 1-ps 1053-nm laser Phys. Rev. Lett. 70 774-7

[12] Krause J, Schafer K and Kulander K 1992 High-order harmonic generation from atoms and ions in the high intensity regime Phys. Rev. Lett. 68 3535-8

[13] Lewenstein M, Balcou Ph, Yu Ivanov M, L'Huillier A and Corkum P B 1994 Theory of high-harmonic generation by low-frequency laser fields Phys. Rev. A 49 2117-32

[14] Pavičić D, Lee K F, Rayner D M, Corkum P B and Villeneuve D M 2007 Direct measurement of the angular dependence of ionization for $\mathrm{N}_{2}, \mathrm{O}_{2}$, and $\mathrm{CO}_{2}$ in intense laser fields Phys. Rev. Lett. 98243001

[15] Kuznetsov A V, Kim C S and Stanton C J 1996 Theory of the generation and detection of carriers in ultrafast pump-andprobe spectroscopy of semiconductors J. Appl. Phys. 805899

[16] Cundiff S T 2014 Optical three dimensional coherent spectroscopy Phys. Chem. Chem. Phys. 16 8193-200

[17] Dwyer J R, Jordan R E, Hebeisen C T, Harb M, Ernstorfer R, Dartigalongue T and Dwayne Miller R J 2007 Femtosecond electron diffraction: an atomic perspective of condensed phase dynamics J. Mod. Optic. 54 905-22

[18] Ruzicka B A and Zhao H 2012 Optical studies of ballistic currents in semiconductors J. Opt. Soc. Am. B 29 A43
[19] Gertsvolf M, Jean-Ruel H, Rajeev P P, Klug D D, Rayner D M and Corkum P B 2008 Orientation-dependent multiphoton ionization in wide band gap crystals Phys. Rev. Lett. 101243001

[20] Schiffrin A et al 2013 Optical-field-induced current in dielectrics Nature 493 70-74

[21] Ghimire S, DiChiara A D, Sistrunk E, Agostini P, DiMauro L F and Reis D A 2010 Observation of high-order harmonic generation in a bulk crystal Nat. Phys. 7 138-41

[22] Schubert O et al 2014 Sub-cycle control of terahertz highharmonic generation by dynamical Bloch oscillations Nat. Photonics 8 119-23

[23] Chalus O, Bates P K, Smolarski M and Biegert J 2009 Mid-IR short-pulse OPCPA with micro-Joule energy at $100 \mathrm{kHz}$ Opt. Express 17 3587-94

[24] Gonze X et al 2009 Computer Phys. Commun. 180 2582-615

[25] Kittel C 1996 Introduction to Solid State Physics (Hoboken, NJ: Wiley)

[26] Savitzky A and Golay M J E 1964 Smoothing and differentiation of data by simplified least squares procedures Anal. Chem. 36 1627-39

[27] Shiner A D, Trallero-Herrero C, Kajumba N, Bandulet H-C, Comtois D, Légaré F, Giguère M, Kieffer J-C,

Corkum P B and Villeneuve D M 2009 Wavelength scaling of high harmonic generation efficiency Phys. Rev. Lett. 103 073902

[28] Shiner A D et al 2013 High harmonic cutoff energy scaling and laser intensity measurement with a $1.8 \mu \mathrm{m}$ laser source. J. Mod. Opt. $601458-65$

[29] Boyd R W 2008 Nonlinear Optics (New York: Academic)

[30] Rayner D M, Naumov A and Corkum P B 2005 Ultrashort pulse non-linear optical absorption in transparent media Opt. Express 133208 